\title{
Studies of Elastic Waves Propagation in Triglycine Sulphate Crystals Doped with Chromium Ions
}

\author{
J. DZIEDZIC \\ Institute of Physics, Technical University of Wrocław \\ Wybrzeże Wyspiańskiego 27, 50-370 Wrocław, Poland
}

(Received July 11, 2001)

\begin{abstract}
Velocities and scattering of transverse elastic wave propagating in the triglycine sulphate crystal doped with the $\mathrm{Cr}^{3+}$ ions as a function of temperature and electric field which polarises the crystal were examined. Temperature dependencies of modulus of elasticity $S_{55}$ and modulus of rigidity $C_{55}$ as a function of temperature and an electric field were determined. The results were analysed and discussed.
\end{abstract}

PACS numbers: $64.70 . \mathrm{Kb}$

\section{Introduction}

The triglycine sulphate (TGS) crystals doped with chromium, Cr, are characterised by the distinct change of electric properties as compared with the pure crystals [1, 2]. In paper [3], the doped crystals are treated as the two-phase systems. One phase consists of a non-perturbed TGS crystal lattice, while the other of spherical domains of the crystal lattice perturbed by aminopyridine molecules (APd). The theory of scattering of longitudinal ultrasonic waves by the non-interacting impurity centres has already been presented in papers [3-7].

However, there are no reports on the influence of the electric field intensity on the elastic properties and on scattering of elastic waves in the TGS crystals doped with the chromium ions $\mathrm{Cr}^{3+}$. This fact has prompted the author to further research.

\section{Experimental}

Resonance frequencies and damping coefficients as a function of temperature and electric field intensity were measured with the use of apparatus whose block 


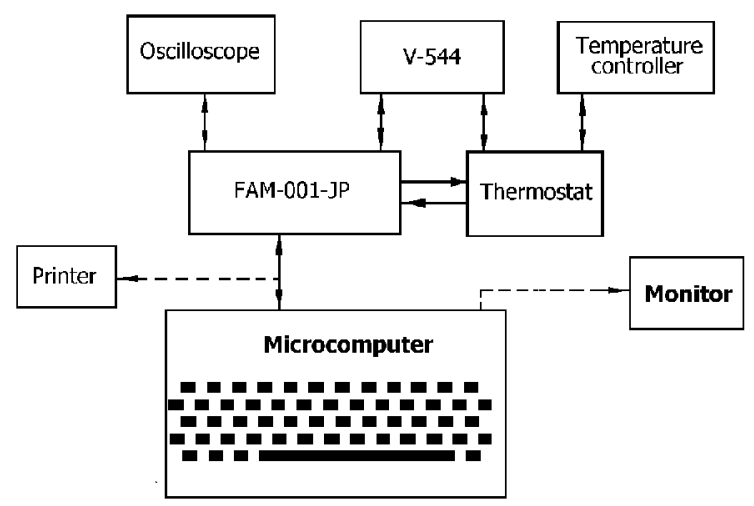

Fig. 1. Block diagram of the measurement system.

diagram is shown in Fig. 1. This measurement system was described in papers [8] and [2]. The measurement error of the damping coefficient does not exceed $1.5 \%$, and of the resonance frequency $0.1 \%$.

For measurement purpose, the TGS samples doped with the chromium ions $\mathrm{Cr}^{3+}$ of $0.5 \%$ concentration which were grown in a ferroelectric phase were used. Crystals were cut perpendicular to the ferroelectric axis in the form of discs with the planar surfaces. On the surfaces perpendicular to the ferroelectric axis the silver electrodes were vapour deposited and then thin, copper wires were glued to these electrodes using silver paste. These wires provided a free suspension of samples in the thermostat and also served as electric connections. So prepared samples were placed in the measuring thermostat and were held at temperature $360 \mathrm{~K}$ for 18 hours to rejuvenate them. Immediately after the rejuvenation the measurements were carried out. The temperature was changed at the rate of $1 \mathrm{~K} / \mathrm{min}$.

The waves were identified by comparison of their resonance frequencies measured at room temperature with the theoretically calculated ones. For the analysis, the transverse wave $f_{13}$ propagating along the $x$-axis and polarised parallel to the $z$-axis was chosen.

\section{Results of examination}

The resonance frequencies and damping coefficients were measured for eight different values of the external electric field intensity which polarised the crystals. Based on the experimental results, the elastic susceptibility modulus $S_{55}$, the rigidity modulus $C_{55}$, and velocities of waves $v_{13}$ were calculated. During calculations the dilatation of the crystals was neglected. The error resulting from this simplification did not exceed $0.94 \%$ in the most unfavourable case.

The relationships between elastic susceptibility modulus $S_{55}$ and elastic rigidity modulus $C_{55}$ as a function of temperature and electric field intensity for the TGS crystals doped with the $\mathrm{Cr}^{3+}$ ions are presented in Figs. 2 and 3. 

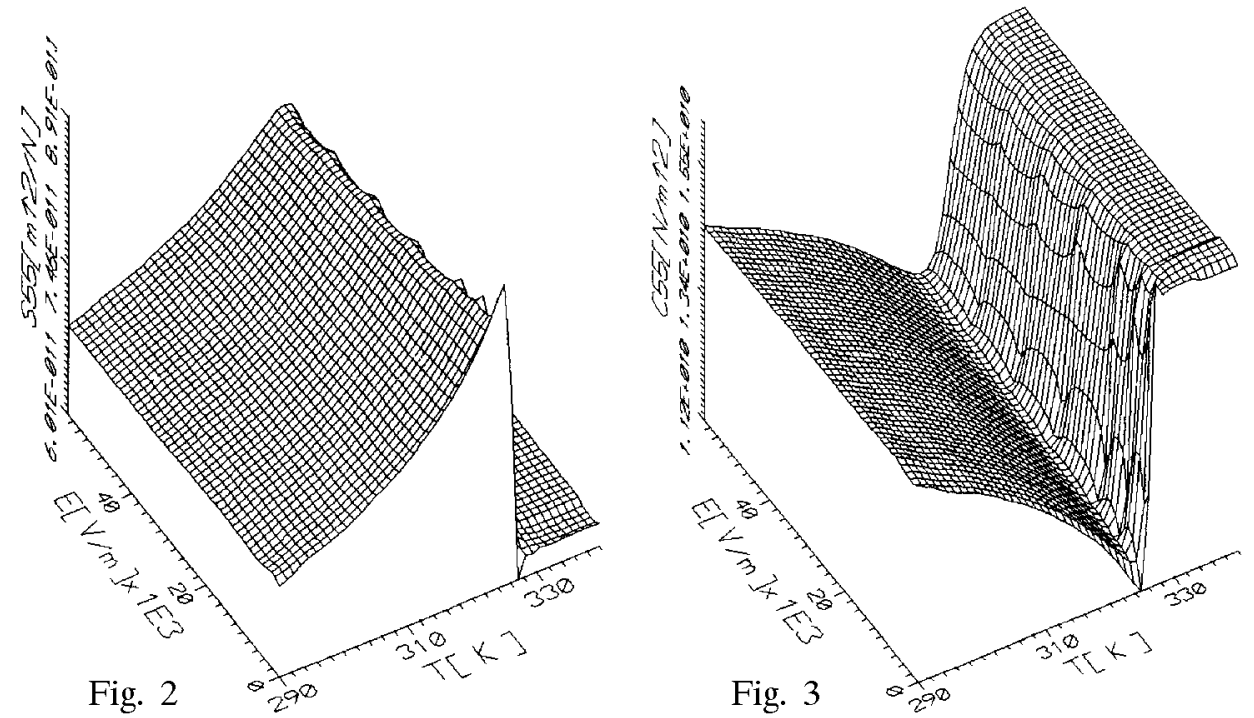

Fig. 2. Elastic susceptibility modulus $S_{55}$ as a function of temperature and intensity of polarising electric field for the TGS crystals doped with the $\mathrm{Cr}^{3+}$ ions at the concentration of $0.5 \%$.

Fig. 3. Elastic rigidity modulus $C_{55}$ as a function of temperature and intensity of polarising electric field for the TGS crystals doped with the $\mathrm{Cr}^{3+}$ ions at the concentration of $0.5 \%$.

Distribution of the velocities of the transverse wave propagating along the $x$-axis and polarised to the $z$-axis of these crystals is shown in Fig. 4 while the damping coefficient of these waves is presented in Fig. 5.

In all cases the electric field causes an increase in the wave velocities in the ferroelectric phase and it was noted that the closer the temperature approaches to the phase transition temperature the more significant influence of the electric field is observed. The polarising field changes the zone of abnormal changes of the wave velocity. This zone is extended toward higher temperatures when the electric field intensity increases. Above the Curie point, all values of velocities of elastic waves propagation increase approaching to some constant values (when the paraelectric mass exceeds significantly the ferroelectric mass in the crystal). When the whole mass of the crystal becomes paraelectric, the piezoelectric properties disappear and further measurements using the method described in this paper are impossible. Hence, the values of wave velocities in the paraelectric phase, just above the phase transition, can be determined by the approximation $v_{13}=f(T)$. These values are constant and independent of the value of the field which polarises the crystal.

The values of the wave velocity $v$ for the TGS crystals doped with $\mathrm{Cr}^{3+}$ ions are lower than those for the pure TGS crystals and larger for the TGS crystals doped with 2-APd [9]. 

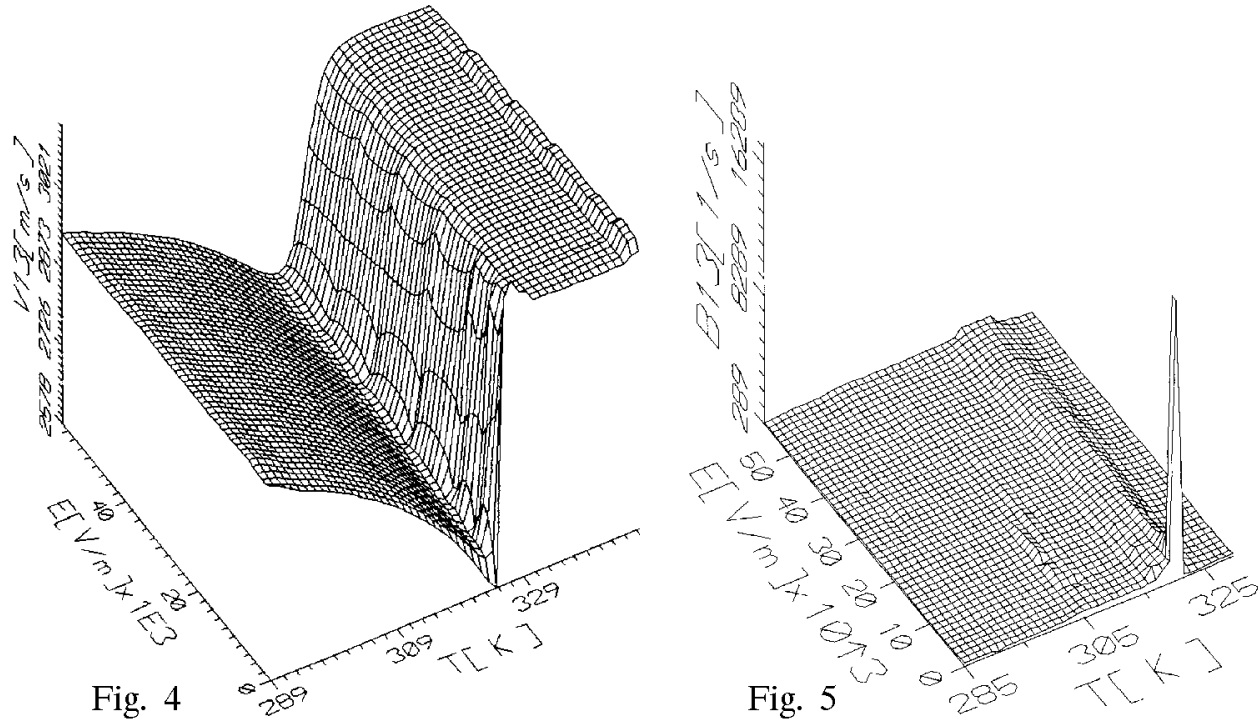

Fig. 4. The velocity of transverse elastic wave $v_{13}$ as a function of temperature and intensity of polarising electric field in the TGS crystals doped with the $\mathrm{Cr}^{3+}$ ions at the concentration of $0.5 \%$.

Fig. 5. Elastic wave damping coefficient $B_{13}$ as a function of temperature and intensity of polarising electric field for the TGS crystals doped with the Cr ions at a concentration of $0.5 \%$.

In each case electric field causes the diminishing of the elastic susceptibility modulus $S$ in the ferroelectric phase, and this change is larger when the temperature of the crystal approaches the phase transition point. Moreover, the electric field causes the expansion of the critical area and its drift towards higher temperature. The values of the modulus $S$ for the TGS crystals doped with the $\mathrm{Cr}^{3+}$ ions are slightly lower than those measured for the crystals doped with 2 -APd and slightly larger than those in the pure TGS crystals [9].

The typical temperature dependencies of the scattering coefficients $B_{13}$ of the elastic waves obtained at different intensities of polarising electric field for the TGS crystals doped with the $\mathrm{Cr}^{3+}$ ions are shown in Fig. 5. From the studies it follows that for the TGS crystals doped with the $\mathrm{Cr}^{3+}$ ions in the ferroelectric phase far from the phase transition temperature the damping coefficient is independent of electric field intensity. On the other hand, the strong influence of the electric field on the value of $B_{13 \max }$ (the maximum value of the damping coefficient in the vicinity of the phase transition temperature) was found. Along with increase in the electric field intensity, the value of $B_{13 \text { max }}$ decreases and simultaneously the half-width of the $B_{13}=f(T)$ curve also increases. The temperature corresponding to $B_{13}$ is shifted toward higher temperatures. 
At the temperatures far from the transition point, the influence of the electric field on the value of $B_{13}$ is negligible but it becomes more and more significant as the temperature approaches $T_{\mathrm{c}}$.

At temperature $300 \mathrm{~K}$, values of $B_{13}$ are equal to $2571 / \mathrm{s}$ for the undoped TGS crystals, $4821 / \mathrm{s}$ for TGS crystals doped with chromium ions $\mathrm{Cr}^{3+}$ and $2431 / \mathrm{s}$ for the TGS crystals doped with 2 -APd. The value of $B_{13 \text { max }}$ (the maximum value of the damping coefficient close to the phase transition temperature) reaches a maximum value for crystals doped with 2 -APd and the minimum value for the TGS crystals doped with the $\mathrm{Cr}^{3+}$ chromium ions [9].

\section{Discussion of the results}

The presented results of investigations of the doped TGS crystals show the distinct increase in the elastic waves velocities, $v$, in the ferroelectric phase with increase in the intensity of polarising electric field along the $b$-axis. No influence of electric field on the approximated values of wave velocities in the paraelectric phase was, however, observed. These facts may be related to the interaction between the electric field and dipole moments of the unit cells. The electric field causes arrangement of the TGS molecules so that they gain some potential energy in this electric field. This energy is added to the energy of crystal bonds, which leads to the growth of the molecular forces. In consequence, we may observe that velocities of the elastic waves increase.

The electric field may also amplify one particular direction of vibrations of ions in a crystal lattice and in effect the principle of equipartition of energy on the individual degrees of freedom may be not fulfilled. This effect rises with the intensity of the electric field. In consequence, a diffusion of the distribution of ion vibration frequency in a crystal lattice takes place which results in a broadening of the region of the abnormal changes of wave velocities coupled with the shift of this region towards higher temperature.

In the paraelectric phase, no dipole moments exist in the unit cells and this is a reason that no influence of the electric field on the elastic wave velocities is observed.

All relationships $B_{13}=f(T)$ are characterised by distinct maxima at the temperatures close to the phase transition point. Such maximum is connected with scattering of mechanical energy during phase transition in a crystal (the fluctuations of the arrangement parameter). Our investigations showed that damping coefficients depend on the nature and the direction of the waves propagating in a crystal. The waves propagating along the $x$-axis and responsible for displacement of molecules along the $z$-axis are the most strongly scattered waves, while the longitudinal waves propagating along the $z$-axis, undergo less distinct scattering [9].

No convincing evidence has been found that in the ferroelectric phase there may exist close relationship between damping coefficients and the electric field 
intensity. However, there is a strong dependence between these quantities in the vicinity of the phase transition point.

A proposal of some explanations may be given taking into consideration the results presented in Ref. [10] related to formation and decay of weak bonds between chains of glycine II, glycine III, and the group of $\mathrm{SO}_{4}^{2-}$ at the temperatures close to $T_{\mathrm{C}}$. Taking into account the statistical character of formation and breaking of these bonds, it may be assumed that probabilities of the formation and breaking of the bonds are described by two different functions of temperature. At each temperature from the critical region an equilibrium between the number of the new formed and broken bonds is established. The number of molecules participating in such processes is an ascending function of temperature. The function reaches a maximum value at a specified temperature and then decreases. The maximum value of the function and its half-width depend on the physical properties of the crystals. As the formation and breaking of bonds lead to non-elastic distortion of the crystal, the energy loss of the propagating elastic wave will be dependent on these distortions. The amount of non-elastic distortions is dependent on the number of molecules participating in such processes at a given temperature.

At the temperature equal to $T_{\mathrm{c}}$, this function reaches its maximum which corresponds to the maximum of the curve $B_{13}=f(T)$.

The electric field causes the temperature dispersion of this number of molecules which participate in the processes of formation and decay of the bonds as well as the shift of their maximum number towards higher temperature with the increase in the electric field intensity. This fact is reflected in the behaviour of the damping coefficients (see Fig. 5).

The increase in the electric field leads to the decrease in the maximum value of damping coefficients and to the increase in the half-width of the curve $B_{13}=f(T)$ at $E=$ const.

\section{References}

[1] H. Wolniewicz, Mol. Sci. 17, 3 (1991).

[2] J. Dziedzic, H. Wolniewicz, Mol. Sci. 18, 41 (1992).

[3] T. Krajewski, J. Dubik, E. Dubik, Fiz. Del. Rad. 8, 189 (1976).

[4] S. Waplak, J. Stankowski, Acta Phys. Pol. 36, 71 (1969).

[5] R. Truell, Phys. Rev. 116, 890 (1959).

[6] C. Ying, R. Truell, J. Appl. Phys. 27, 1086 (1956).

[7] J. Malecki, Teoria fal i układów akustycznych, PWN, Warszawa 1964.

[8] J. Dziedzic, Ph.D. thesis, Technical University of Wrocław, Wrocław 1979.

[9] J. Dziedzic, H. Wolniewicz, Mol. Sci. 19, 35 (1993/5).

[10] J. Stankowski, Ferroelectrics 28, 107 (1978). 